\title{
High Resolution Photoelectron Spectroscopy of Vibrationally Excited $\mathrm{OH}^{-}$
}

\author{
Martin DeWitt, ${ }^{\mathrm{a}, \S}$ Mark C. Babin, ${ }^{\mathrm{a}, \S}$ Daniel M. Neumark ${ }^{\mathrm{a}, \mathrm{b} *}$ \\ ${ }^{a}$ Department of Chemistry, University of California, Berkeley, CA 94720, USA \\ ${ }^{b}$ Chemical Sciences Division, Lawrence Berkeley National Laboratory, Berkeley, CA 94720, USA.
}

$\S$ These authors contributed equally.

\section{Corresponding Author}

* dneumark@berkeley.edu

\begin{abstract}
The effect of vibrational pre-excitation of anions on their photoelectron spectra is explored, combining slow photoelectron velocity-map imaging of cryogenically cooled anions (cryo-SEVI) with tunable IR radiation to pre-excite the anions. This new IR cryo-SEVI method is applied to $\mathrm{OH}^{-}$as a test system, where the $\mathrm{R}(0)$ transition of the hydroxyl anion $\left(3591.53 \mathrm{~cm}^{-1}\right)$ is pumped. Vibrational excitation induces a $30 \%$ depletion in photodetachment signal from the $v=0, J=0$ ground state of the anion and the appearance of all five allowed, rotationally-resolved photodetachment transitions from the $\mathrm{OH}^{-}(v=1$, $J=1$ ) level, each with peak widths between 1-2 $\mathrm{cm}^{-1}$. By scanning the IR laser, IR cryo-SEVI can also serve as a novel action technique to obtain the vibrational spectrum of $\mathrm{OH}^{-}$, giving an experimental value for the $\mathrm{R}(0)$ transition of $3591(1.2) \mathrm{cm}^{-1}$.
\end{abstract}

\section{Introduction}

Anion photoelectron spectroscopy (PES) is a powerful and versatile method for determining electron affinities, the vibrational and electronic energy levels of neutral species created by photodetachment, and the geometric changes that occur upon photodetachment. ${ }^{1-6}$ It has been applied with considerable success to free radicals, clusters, and reaction transition states. ${ }^{6-9}$ In favorable circumstances, anion PES yields resolved vibrational structure. The extent of this vibrational structure is generally governed by Franck-Condon considerations; only totally symmetric vibrational modes in which there is a normal coordinate displacement upon photodetachment are active in the PE spectrum. Hence, for example, one does not typically observe vibrational motion involving free $\mathrm{OH},{ }^{10} \mathrm{NH},{ }^{11}$ and $\mathrm{CH}^{12}$ bonds since the anion and neutral bond lengths change very little upon photodetachment. Likewise, transitions involving non-totally symmetric modes are weak; odd $\Delta v$ transitions in such modes are symmetryforbidden in the absence of vibronic coupling, ${ }^{13-14}$ and even $\Delta v$ transitions require a large frequency change to gain appreciable intensity in a photoelectron spectrum. ${ }^{15}$ However, if the anion is vibrationally excited, new vibrational levels of the neutral become accessible by photodetachment. The effects of vibrational hot bands on the photoelectron spectrum of the vinylidene anion $\left(\mathrm{H}_{2} \mathrm{CC}^{-}\right)$were considered by Johnson, ${ }^{16}$ while Continetti and Guo showed that vibrational pre-excitation of the $\mathrm{F}^{-}\left(\mathrm{H}_{2} \mathrm{O}\right)$ anion enables one to access otherwise inaccessible regions of the $\mathrm{F}+\mathrm{H}_{2} \mathrm{O}$ potential energy surface. ${ }^{17} \mathrm{In}$ this paper, we explore the effect of infrared laser excitation of an anion, $\mathrm{OH}^{-}$, upon its photoelectron spectrum.

The hydroxyl radical and corresponding anion have a rich spectroscopic history. The microwave spectrum of the radical was measured by Townes in $1955,{ }^{18}$ and since then the high resolution spectroscopy of this species has been mapped out in great detail. ${ }^{19-21} \mathrm{OH}$ has a ${ }^{2} \Pi$ ground state with a spin-orbit splitting of $126.2923 \mathrm{~cm}^{-1}$ between the $\Omega=3 / 2$ and $1 / 2$ fine structure levels and a rotational constant, $\mathrm{B}_{\mathrm{e}}$, of $18.91083(106) \mathrm{cm}^{-1} .^{22}$ 
The hydroxide anion has likewise been investigated by a series of state-of-the-art spectroscopic techniques. Rotationally resolved photodetachment transitions were observed in a series of high resolution laser photodetachment threshold measurement experiments by Lineberger ${ }^{23-26}$ and, more recently, by Wester. ${ }^{27-28}$ The rotationally resolved infrared spectrum of $\mathrm{OH}^{-}$was measured by Saykally using velocity-modulation spectroscopy ${ }^{29}$ and by Schlemmer via laser-induced reactivity (LIR) in a cold ion trap. ${ }^{30}$ Blondel used photodetachment microscopy to obtain well-resolved rotational transitions between $\mathrm{OH}^{-}$and the fine structure levels of $\mathrm{OH}$ and determined a refined rotational constant of $18.7352(3) \mathrm{cm}^{-1}$ for the anion. ${ }^{10}$

Here, we combine slow electron velocity-map imaging of cryogenically cooled $\mathrm{OH}^{-}$(cryo$\mathrm{SEVI})^{31}$ with infrared pre-excitation to investigate the effect of vibrational excitation upon its photoelectron spectrum. Cryo-SEVI is a high-resolution variant of photoelectron spectroscopy in which cryogenically cooled anions are photodetached with a tunable laser, and the resulting photoelectrons are analyzed using a velocity-map imaging system designed specifically to measure the kinetic energies of slower electrons with greater precision. ${ }^{6,31}$ The combination of cryogenic cooling and slow-electron imaging yields photoelectron spectra with energy resolution as high as $1-2 \mathrm{~cm}^{-1}$, and in the case of $\mathrm{OH}^{-}$, we obtain well-resolved rotational features. Upon infrared pre-excitation, the IR cryo-SEVI spectrum reveals new rotationally resolved features resulting from photodetachment transitions between the $v=1$ levels of $\mathrm{OH}^{-}$and $\mathrm{OH}$.

\section{Experimental Methods}

The cryo-SEVI method has been previously described in detail. ${ }^{6,31-32}$ In this work, $\mathrm{O}^{-}$anions are generated by expanding a dilute gas mixture of $\mathrm{N}_{2} \mathrm{O}$ in helium backing gas through a pulsed Even-Lavie valve, ${ }^{33}$ equipped with a circular filament ionizer. Electrons from the ionizer induce dissociative attachment of $\mathrm{N}_{2} \mathrm{O}$ to produce $\mathrm{O}^{-}$. The ions are directed through a radiofrequency $(\mathrm{RF})$ hexapole ion guide, a RF quadrupole mass filter, and into a cryogenically cooled linear RF octupole ion trap held at $5 \mathrm{~K}$. The ions are stored in the trap for $\sim 40 \mathrm{~ms}$, where they are collisionally cooled with an 80:20 He: $\mathrm{H}_{2}$ buffer gas mixture to their ground electronic state. Inside the trap, $\mathrm{O}^{-}$anions react with $\mathrm{H}_{2}$ molecules to form $\mathrm{OH}^{-}$anions, which are rotationally and vibrationally cooled by further collisions with the buffer gas.

The ions are extracted from the trap into an orthogonal Wiley-McLaren time-of-flight mass spectrometer and guided into the laser interaction region of a seven-plate velocity-map imaging (VMI) spectrometer. ${ }^{34-35}$ Vertically polarized light from a dye laser pumped by the second harmonic of a $\mathrm{Nd}$ :YAG laser operating at $20 \mathrm{~Hz}$ is focused to a spot size of $\sim 0.01 \mathrm{~mm}^{2}$ and used to photodetach $\mathrm{OH}^{-}$ anions. The dye laser system can produce radiation between $220 \mathrm{~nm}$ and $1.8 \mu \mathrm{m}$ with a spectral bandwidth of $<0.1 \mathrm{~cm}^{-1}$ and pulse energies up to $15 \mathrm{~mJ}$. The resulting photoelectrons are projected onto a $2 \mathrm{D}$ detector that comprises two chevron-stacked microchannel plates coupled to a phosphor screen. ${ }^{36} \mathrm{~A}$ CCD camera photographs the phosphor screen after each laser shot, and the centroids of the photoelectron events in each image are calculated and binned in a $1024 \times 1024$ grid in real time. ${ }^{37}$

Several modifications to the cryo-SEVI technique are required to facilitate the selective vibrational excitement of cryogenically-cooled anions using infrared radiation prior to photodetachment (IR cryo-SEVI). On alternating experimental cycles, anions are vibrationally excited prior to photodetachment using the horizontally polarized mid-infrared (mIR) output of a tabletop OPO/OPA laser system (LaserVision), pumped by a Nd:YAG laser (Continuum SureLite EX) operating at $10 \mathrm{~Hz}$. The infrared (IR) laser system can produce radiation between $1.3 \mu \mathrm{m}$ and $16 \mu \mathrm{m}$ with a spectral bandwidth of $\sim 3.5 \mathrm{~cm}^{-1}$ and pulse energies up to $4 \mathrm{~mJ}{ }^{38}$ The mIR beam is rotated to ensure vertical polarization in the interaction region and focused to a spot size of $\sim 0.07 \mathrm{~mm}^{2}$. It is aligned to be collinear and 
counterpropagating to the visible beam from the dye laser. The IR laser system is triggered such that the mIR light excites the ion packet less than $1 \mathrm{~ns}$ before photodetachment.

Photoelectron centroids are accumulated over several thousand experimental cycles into two VMI images, depending on whether the IR laser system was triggered in that cycle, allowing for photodetachment images with and without IR pre-excitation to be acquired simultaneously. The radial and angular photoelectron distributions are reconstructed from each accumulated image using the Maximum Entropy Velocity Legendre Reconstruction (MEVELER) method. ${ }^{39}$ The electron kinetic energy (eKE) distributions are related to the radial distributions by acquiring images of the well-characterized detachment transitions of $\mathrm{O}^{-}$at several different photon energies. ${ }^{40}$

Due to the roughly constant resolving power $(\triangle \mathrm{eKE} / \mathrm{eKE})$ of the VMI spectrometer, slow photoelectrons give the highest resolution features. ${ }^{34-35}$ For this reason, cryo-SEVI and IR cryo-SEVI spectra are acquired by first taking an overview spectrum at a relatively high photon energy before tuning the detachment laser to energies slightly above features of interest. Narrow windows surrounding the high-resolution features are concatenated together and scaled to match the relative intensities of the overview spectrum, resulting in a single high-resolution photoelectron spectrum. Cryo-SEVI and IR cryoSEVI spectra are plotted against electron binding energy (eBE), given by $e B E=h v-e K E$. A difference spectrum is also calculated by subtracting the eKE distributions with and without IR pre-excitation.

\section{Results and Discussion}

The cryo-SEVI and IR cryo-SEVI spectra of $\mathrm{OH}^{-}$are presented in Figure 1. Fig. 1a shows the spectrum with no IR excitation, Fig. 1b shows the vibrationally excited IR cryo-SEVI spectrum with the IR laser tuned to the $\mathrm{R}(0)$ rovibrational transition of $\mathrm{OH}^{-}$at $3591 \mathrm{~cm}^{-1},{ }^{29}$ and Fig. 1c shows the difference spectrum. In all three scans, the black traces are high resolution scans taken at several photon energies slightly above threshold for each feature. The blue trace in Fig. 1a is a low-resolution overview scan taken at a relatively high photon energy. The red traces in Fig. $1 \mathrm{~b}$ are from high-resolution scans taken with the IR laser on and represent either new or depleted features resulting from IR excitation.

As discussed previously, ${ }^{10,25,27}$ the near-threshold photodetachment of $\mathrm{OH}^{-}$is dominated by swave detachment with electron orbital angular momentum $l=0$. Combining this with the unit angular momentum provided by the photon results in a photodetachment rotational selection rule of $J^{\prime}-J^{\prime \prime}=\Delta J=$ $\pm 1 / 2, \pm 3 / 2$, where $J^{\prime}$ and $J^{\prime \prime}$ are the total angular momentum quantum numbers of the neutral and negative ion, respectively. Due to the change in spin between anion and neutral, it is more convenient to use the quantum number $N=J-S$ (total angular momentum without spin) when considering transitions between rotational levels. For $\mathrm{OH}^{-}, N^{\prime \prime}=J^{\prime \prime}$, while in the ${ }^{2} \Pi_{3 / 2}\left({ }^{2} \Pi_{1 / 2}\right)$ state of $\mathrm{OH}, N^{\prime}=J^{\prime}-1 / 2\left(N^{\prime}=J^{\prime}+{ }^{1} / 2\right)$. Detachment transitions are labeled $\mathrm{O}, \mathrm{P}, \mathrm{Q}, \mathrm{R}$, and $\mathrm{S}$ indicating $\Delta N$ of $-2,-1,0,+1$, and +2 , respectively. The rotational selection rule $\Delta J= \pm 1 / 2, \pm^{3} / 2$ dictates that transitions in the $\mathrm{S}$ branch of the ${ }^{2} \Pi_{3 / 2}$ manifold and transitions in the $\mathrm{O}$ branch of the ${ }^{2} \Pi_{1 / 2}$ manifold are formally forbidden; similar selection rules were seen in the anion zero electron kinetic energy (ZEKE) spectrum of $\mathrm{SH}^{-} .{ }^{41}$ Following Goldfarb et al, ${ }^{10}$ transitions to $\Omega^{\prime}=3 / 2$ and $1 / 2$ levels of $\mathrm{OH}$ are labeled in Fig. 2 with indices of 3 and 1 , respectively. The R3(0) transition, for example, corresponds to detachment from the $N^{\prime \prime}=0$ state of the anion to the $N^{\prime}=1$ $\left(J^{\prime}=3 / 2\right)$ state of the $\Omega^{\prime}=3 / 2$ series in the neutral.

The cryo-SEVI and IR cryo-SEVI spectra (Figs. 1a and 1b) exhibit a number of well-resolved transitions to both of the spin-orbit states of the neutral radical, with typical peak widths of $2-5 \mathrm{~cm}^{-1}$ FWHM. Peak assignments are given in Table 1, with rotational transitions as indicated in Fig. 2 and vibrational transitions labeled as $v^{\prime}-v^{\prime \prime}$, where $v^{\prime}$ and $v^{\prime}$ are the vibrational quantum numbers in the neutral and anion, respectively. The previously reported transition energies in Table 1 were determined by 
computing the expected shift in energy of the R3(0) and R1(0) transitions, reported by Goldfarb et al., ${ }^{10}$ based on the energy spacings between known rotational levels in $\mathrm{OH}^{-}$and $\mathrm{OH}$, given by Rosenbaum et $a l .{ }^{29}$ and Mélen et $a l,{ }^{21}$ respectively.

The largest feature in Fig. 1a, labeled $\mathrm{G}_{3}$, is the R3(0) transition between the lowest vibrationrotation levels of $\mathrm{OH}^{-}$and $\mathrm{OH}$, thereby yielding an electron affinity of the $\mathrm{OH}$ radical of $14741(2) \mathrm{cm}^{-1}$, in good agreement with that reported by Goldfarb et al. ${ }^{10}$ Beyond this, the spectrum reveals two overlapping sets of peaks, $(\mathrm{A}-\mathrm{H})_{3}$ and $(\mathrm{A}-\mathrm{I})_{1}$, that correspond to detachment to different rotational levels of the ${ }^{2} \Pi_{3 / 2}$ and ${ }^{2} \Pi_{1 / 2}$ states of neutral $\mathrm{OH}$, respectively. Many of the features observed in the cryo-SEVI spectrum have been previously reported by Schulz et al. ${ }^{25}$ and Goldfarb et al, ${ }^{10}$ allowing for their direct assignment. Features (A-C) $)_{3}$ correspond to the P3 branch, features (D-F) $)_{3}$ correspond to the $\mathrm{Q} 3$ branch, $(\mathrm{G}-\mathrm{H})_{3}$ correspond to the $\mathrm{R} 3$ branch, $(\mathrm{A}-\mathrm{C})_{1}$ correspond to the $\mathrm{Q} 1$ branch, and $(\mathrm{D}-\mathrm{F})_{1}$ and $\mathrm{H}_{1}$ correspond to the R1 branch. We clearly observe the weak $\mathrm{S} 1(0)$ and $\mathrm{S} 1(1)$ features $\mathrm{G}_{1}$ and $\mathrm{I}_{1}$, in previous work, ${ }^{10}$ only the S1(0) transition was reported, and that was barely visible.

While there are a few features in the cryo-SEVI spectrum originating from anion rotational levels with $\mathrm{J}>2$, the most intense features arise from transitions from the $J=0$ and 1 levels, indicating the ions are rotationally cold. The rotational temperature of the anions can be estimated by fitting the ratio of the intensities of the R3(0) and R3(1) features to a Boltzmann rotational distribution, given by

$$
\frac{I_{1}}{I_{0}}=3 e^{-2 h c B_{0} / k_{b} T}
$$

where $I_{0}$ and $I_{l}$ are the intensities of the R3(0) and R3(1) peak, respectively, $h$ is Planck's constant, $c$ is the speed of light, $B_{0}$ is the rotational constant of $\mathrm{OH}^{-}\left(18.7354(16) \mathrm{cm}^{-1}\right)^{10}, k_{b}$ is Boltzmann's constant, and $T$ is the rotational temperature of the anion. When comparing the intensities of these two peaks at a photodetachment energy of $666.48 \mathrm{~nm}$, a rotational temperature of roughly $25 \mathrm{~K}$ is estimated, which is only slightly higher in temperature than the cryogenic trap and similar to rotational temperatures of $\mathrm{OH}^{-}$ measured by Otto et al. while held in a 22-pole RF ion trap held at $8 \mathrm{~K}^{27}$

In the IR cryo-SEVI spectrum (Fig. 1b), the $\mathrm{R}(0)$ rovibrational transition of $\mathrm{OH}^{-}$anions was pumped using $2.78 \mu \mathrm{m}\left(3591 \mathrm{~cm}^{-1}\right)$ light on alternating experimental cycles prior to photodetachment, forming anions in the $\left(v^{\prime \prime}, J^{\prime}\right)=(1,1)$ rovibrational level. In these spectra, there is a clear reduction in the relative intensities of peaks $\mathrm{G}_{3}$ and $\mathrm{D}_{1}$, indicating a $\sim 30 \%$ depletion of the $(0,0)$ population of the detached anions. Conversely, five new features appear $\left(\mathrm{d}_{3}, \mathrm{~h}_{3}, \mathrm{c}_{1}, \mathrm{e}_{1}\right.$ and $\left.\mathrm{i}_{1}\right)$ and correspond well with the calculated values for detachment from the $(1,1)$ rovibrational level of $\mathrm{OH}^{-}$to the $\left(v^{\prime}, \mathrm{J}^{\prime}\right)=\left(1,{ }^{3} / 2\right)$ and $(1,5 / 2)$ levels in the ${ }^{2} \Pi_{3 / 2}$ state and to the $(1,1 / 2),(1,3 / 2)$, and $(1,5 / 2)$ levels in the ${ }^{2} \Pi_{1 / 2}$ state. These, in fact, are all of the expected transitions from a $J "=1$ rotational level in the anion based on the $\Delta J= \pm 1 / 2, \pm^{3} / 2$ selection rule. Each appears within $15 \mathrm{~cm}^{-1}$ of a nearby ground-state transition due to the slight difference in vibrational frequency between the anion and neutral, demonstrating the necessity for a high-resolution technique, such as cryo-SEVI, to resolve these features. Note that although the $0-1$ hot band photodetachment transition would be well separated from the ground state SEVI transitions, the FranckCondon factor for this transition is roughly four orders of magnitude smaller than that for the $0-0$ transition, hence observation of $0-1$ rovibrational transitions via cryo-SEVI would be exceptionally challenging.

Fig. 1c shows the IR cryo-SEVI difference spectrum, calculated by subtracting the photoelectron distribution of the cryo-SEVI spectrum from that of the IR cryo-SEVI spectrum and normalizing to the $\mathrm{G}_{3}$ depletion feature. Transitions arising from vibrationally excited anions in the IR cryo-SEVI spectrum 
appear as positive features in the difference spectrum, while transitions from states depleted by vibrational excitation appear as negative features. As the cryo-SEVI and IR cryo-SEVI spectra are acquired simultaneously, experimental variations in ion signal or detachment laser power should affect both spectra identically, greatly simplifying the comparison of these data and the generation of difference spectra. However, slight variations are still present, which can lead to peaks from the non-excited spectrum appearing in the difference spectrum, such as peak $E_{1}$ appearing as a positive peak, as well as causing noise in the difference spectrum that could be mistaken for very weak signal - this is most prevalent in the high eBE range of Fig. 1c, where the weak feature $i_{1}$ is comparable in intensity to noise.

IR cryo-SEVI can also be used as an infrared spectroscopic technique, capable in principle of determining the vibrational spectra of cryogenically cooled anions. Typically, anion vibrational frequencies are mapped out with action spectroscopic techniques in which anions are complexed with weakly bound "tags" such as Ar or $\mathrm{D}_{2}$ that are shed when the anion is vibrationally excited. ${ }^{42-43}$ These tags can result in small perturbations of the anion vibrational frequencies. IR cryo-SEVI circumvents this effect by instead scanning the output of the OPO/OPA while monitoring the electron signal - a maximum in the sequence band intensity in the cryo-SEVI spectrum should be achieved when the IR frequency matches the transition energy. This is shown in Fig. 3a, which displays representative IR cryo-SEVI spectra in this IR scanning mode where feature $h_{3}$ grows in as the photon energy is scanned through the $\mathrm{R}(0)$ transition in $\mathrm{OH}^{-}$. The uncertainty in the anion vibrational frequency determined by this method can be related to the intensity of the feature as a function of IR wavelength - plotting the integrated area of $h_{3}$ as a function of energy and fitting it to a Gaussian distribution, as is plotted in Fig. 3b, returns a FWHM of $2.80 \mathrm{~cm}^{-1}$, comparable to the reported bandwidth of the LaserVision output. One-sigma errors in the reported vibrational frequency are then $1.2 \mathrm{~cm}^{-1}$. In the case of $\mathrm{OH}^{-}$, we find the $\mathrm{R}(0)$ rovibrational transition energy to be $3591(1.2) \mathrm{cm}^{-1}$, encompassing the value of $3591.5254 \mathrm{~cm}^{-1}$ measured by Rosenbaum et al. ${ }^{29}$ The six IR cryo-SEVI spectra used to generate the IR spectrum in Fig. 3b were each accumulated for around 20,000 experimental cycles, equating to roughly 1.5 hours of data collection in total. Compared to infrared photodissociation of tagged anions, the necessity of obtaining a reasonably high quality photoelectron spectrum at each IR wavelength limits the utility of IR cryo-SEVI as a means of obtaining an infrared spectrum over a large frequency range, but improved collection and data analysis methods may narrow this gap in the near future.

The large rotational constant of the hydroxide anion made it possible to monitor the effect of IR excitation on specific rotational transitions in the cryo-SEVI spectrum, which would typically not be feasible for polyatomic systems. However, in our experience, the rotational profiles of cryo-cooled anions are around $5 \mathrm{~cm}^{-1}$ wide, ${ }^{6}$ which matches well with the $3 \mathrm{~cm}^{-1}$ bandwidth of the IR laser system. It should thus be possible to vibrationally excite most of the anion population and observe new vibrational features in the photoelectron spectrum from transitions to neutral vibrational levels that are inaccessible from the anion ground state. One can, for example, excite IR active, non-totally symmetric modes in the anion, as this will enable one to access normally inactive, non-totally symmetric modes of the neutral species. Such an experiment would be of particular interest for the vinylidene anion $\mathrm{H}_{2} \mathrm{CC}^{-}$, where it has been shown that excitation of non-totally symmetric modes of neutral $\mathrm{H}_{2} \mathrm{CC}$ drives isomerization to $\mathrm{HCCH} .{ }^{44}$ This experiment will be facilitated by previously measured and calculated vibrational frequencies for the vinylidene anion. ${ }^{16,45}$ Overall, the range of applicability of IR cryo-SEVI remains to be determined, but any anion with a relatively isolated vibrational origin transition in its cryo-SEVI spectrum is a viable candidate.

\section{Conclusion}


We introduce a new technique, slow photoelectron velocity-map imaging of cryogenically cooled anions with selective vibrational excitation (IR cryo-SEVI), which combines infrared vibrational excitation with the high photoelectron energy resolution of cryo-SEVI. The efficacy of this technique is demonstrated on a diatomic, $\mathrm{OH}^{-}$, through the appearance of new features in the photoelectron spectrum after vibrational excitation, corresponding to all observable photodetachment transitions from the $v=1, J$ $=1$ level of the anion. These results highlight the capabilities of IR cryo-SEVI to probe unexplored regions of neutral potential energy surfaces via selective addition of sequence bands along normal coordinates that would normally be inactive owing to Franck-Condon considerations, as well as a new action technique capable of collecting vibrational spectra of anions with $\sim 1 \mathrm{~cm}^{-1}$ resolution.

\section{Acknowledgments}

This research was funded by the Air Force Office of Scientific Research under Grant No. FA9550-19-10051, as well as through AFOSR's Defense University Research Instrumentation Program under Grant No. FA9550-19-1-0139, DEF. M. C. B. thanks the Army Research Office for a National Defense Science and Engineering Graduate fellowship.

\section{References}

1. Ellison, G. B.; Engelking, P. C.; Lineberger, W. C., Experimental-Determination of Geometry and Electron-Affinity of $\mathrm{CH}_{3} . J$. Am. Chem. Soc. 1978, 100, 2556-2558.

2. Rienstra-Kiracofe, J. C.; Tschumper, G. S.; Schaefer, H. F.; Nandi, S.; Ellison, G. B., Atomic and molecular electron affinities: Photoelectron experiments and theoretical computations. Chem. Rev. 2002, 102, 231-282.

3. $\quad$ Simons, J., Molecular anions. J. Phys. Chem. A 2008, 112, 6401-6511.

4. Ichino, T.; Wren, S. W.; Vogelhuber, K. M.; Gianola, A. J.; Lineberger, W. C.; Stanton, J. F., The vibronic level structure of the cyclopentadienyl radical. J. Chem. Phys. 2008, 129, 084310.

5. Lineberger, W. C., Once upon Anion: A Tale of Photodetachment. Annu. Rev. Phys. Chem. 2013, 64, 21-36.

6. Weichman, M. L.; Neumark, D. M., Slow Photoelectron Velocity-Map Imaging of Cryogenically Cooled Anions. Annu. Rev. Phys. Chem. 2018, 69, 101-124.

7. Leopold, D. G.; Ho, J.; Lineberger, W. C., Photoelectron-Spectroscopy of Mass-Selected Metal Cluster Anions. I. $\mathrm{Cu}_{\mathrm{n}}^{-}, \mathrm{n}=1-10$. J. Chem. Phys. 1987, 86, 1715-1726.

8. Castleman, A. W.; Bowen, K. H., Clusters: Structure, energetics, and dynamics of intermediate states of matter. J. Phys. Chem. 1996, 100, 12911-12944.

9. Continetti, R. E.; Guo, H., Dynamics of transient species via anion photodetachment. Chem. Soc. Rev. 2017, 46, 7650-7667.

10. Goldfarb, F.; Drag, C.; Chaibi, W.; Kroger, S.; Blondel, C.; Delsart, C., Photodetachment microscopy of the $\mathrm{P}, \mathrm{Q}$, and $\mathrm{R}$ branches of the $\mathrm{OH}^{-}(\mathrm{v}=0)$ to $\mathrm{OH}(\mathrm{v}=0)$ detachment threshold. $J$. Chem. Phys. 2005, 122, 014308.

11. Engelking, P. C.; Lineberger, W. C., Laser Photoelectron Spectrometry of $\mathrm{NH}^{-}$: Electron-affinity and Intercombination Energy Difference in NH. J. Chem. Phys. 1976, 65, 4223-4224.

12. Kasdan, A.; Herbst, E.; Lineberger, W. C., Laser Photoelectron Spectrometry of CH. Chem. Phys. Lett. 1975, 31, 78-82.

13. Ervin, K. M.; Lineberger, W. C., Photoelectron-spectra of $\mathrm{C}_{2}^{-}$and $\mathrm{C}_{2} \mathrm{H}^{-}$. J. Phys. Chem. 1991, 95, 1167-1177.

14. Weaver, A.; Arnold, D. W.; Bradforth, S. E.; Neumark, D. M., Examination of the ${ }^{2} \mathrm{~A}^{\prime}{ }_{2}$ and ${ }^{2} \mathrm{E}^{6}$ states of $\mathrm{NO}_{3}$ by ultraviolet photoelectron spectroscopy of $\mathrm{NO}_{3}^{-}$. J. Chem. Phys. 1991, 94, 1740-1751. 
15. Herzberg, G., Electronic spectra and electronic structure of polyatomic molecules. Van Nostrand: New York, 1966; Vol. 3.

16. Gerardi, H. K.; Breen, K. J.; Guasco, T. L.; Weddle, G. H.; Gardenier, G. H.; Laaser, J. E.; Johnson, M. A., Survey of Ar-Tagged Predissociation and Vibrationally Mediated Photodetachment Spectroscopies of the Vinylidene Anion, $\mathrm{C}_{2} \mathrm{H}_{2}^{-}$. J. Phys. Chem. A 2010, 114, 1592-1601.

17. Ray, A. W.; Ma, J. Y.; Otto, R.; Li, J.; Guo, H.; Continetti, R. E., Effects of vibrational excitation on the $\mathrm{F}+\mathrm{H}_{2} \mathrm{O}->\mathrm{HF}$ plus $\mathrm{OH}$ reaction: dissociative photodetachment of overtone-excited $\mathrm{F}-\mathrm{H}-\mathrm{OH}^{-}$. Chem. Sci. 2017, 8, 7821-7833.

18. Dousmanis, G. C.; Sanders, T. M.; Townes, C. H., Microwave Spectra of the Free Radicals OH and OD. Phys. Rev. 1955, 100, 1735-1755.

19. Beaudet, R. A.; Poynter, R. L., Microwave-Spectra of Molecules of Astrophysical Interest XII. Hydroxyl Radical. J. Phys. Chem. Ref. Data 1978, 7, 311-362.

20. Coxon, J. A., Optimum Molecular Constants and Term Values for the $X^{2} \Pi(v \leq 5)$ and $A^{2} \Sigma^{+}(v \leq$ 3) States of OH. Can. J. Phys. 1980, 58, 933-949.

21. Melen, F.; Sauval, A. J.; Grevesse, N.; Farmer, C. B.; Servais, C.; Delbouille, L.; Roland, G., A new analysis of the $\mathrm{OH}$ radical spectrum from solar infrared observations. J. Mol. Spectrosc. 1995, 174, 490-509.

22. Maillard, J. P.; Chauville, J.; Mantz, A. W., High-resolution emission spectrum of OH in an oxyacetylene flame from 3.7 to $0.9 \mu \mathrm{m}$. J. Mol. Spectrosc. 1976, 63, 120-141.

23. Hotop, H.; Patterson, T. A.; Lineberger, W. C., High-Resolution Photodetachment Study of $\mathrm{OH}^{-}$ and $\mathrm{OD}^{-}$in Threshold Region 7000-6450 A. J. Chem. Phys. 1974, 60, 1806-1812.

24. Breyer, F.; Frey, P.; Hotop, H., High-Resolution Photoelectron Spectrometry of Negative-Ions Rotational Transitions in Laser-Photodetachment of $\mathrm{OH}^{-}, \mathrm{SH}^{-}, \mathrm{SD}^{-}$. Z Phys a-Hadron Nucl 1981, 300, 7 -

24.

25. Schulz, P. A.; Mead, R. D.; Jones, P. L.; Lineberger, W. C., $\mathrm{OH}^{-}$and $\mathrm{OD}^{-}$Threshold

Photodetachment. J. Chem. Phys. 1982, 77, 1153-1165.

26. Smith, J. R.; Kim, J. B.; Lineberger, W. C., High-resolution threshold photodetachment spectroscopy of $\mathrm{OH}^{-}$. Phys. Rev. A 1997, 55, 2036-2043.

27. Otto, R.; Von Zastrow, A.; Best, T.; Wester, R., Internal state thermometry of cold trapped molecular anions. Phys. Chem. Chem. Phys. 2013, 15, 612-618.

28. Lakhmanskaya, O.; Simpson, M.; Wester, R., Vibrational overtone spectroscopy of cold trapped hydroxyl anions. Phys. Rev. A 2020, 102, 012809.

29. Rosenbaum, N. H.; Owrutsky, J. C.; Tack, L. M.; Saykally, R. J., Velocity Modulation Laser Spectroscopy of Negative-Ions - the Infrared-Spectrum of Hydroxide $\left(\mathrm{OH}^{-}\right)$. J. Chem. Phys. 1986, 84, 5308-5313.

30. Jusko, P.; Asvany, O.; Wallerstein, A. C.; Brunken, S.; Schlemmer, S., Two-Photon Rotational Action Spectroscopy of Cold $\mathrm{OH}^{-}$at 1 ppb Accuracy. Phys. Rev. Lett. 2014, 112, 253005.

31. Hock, C.; Kim, J. B.; Weichman, M. L.; Yacovitch, T. I.; Neumark, D. M., Slow photoelectron velocity-map imaging spectroscopy of cold negative ions. J. Chem. Phys. 2012, 137, 244201.

32. Osterwalder, A.; Nee, M. J.; Zhou, J.; Neumark, D. M., High resolution photodetachment spectroscopy of negative ions via slow photoelectron imaging. J. Chem. Phys. 2004, 121, 6317-6322.

33. Even, U.; Jortner, J.; Noy, D.; Lavie, N.; Cossart-Magos, C., Cooling of large molecules below 1 $\mathrm{K}$ and He clusters formation. J. Chem. Phys. 2000, 112, 8068-8071.

34. Eppink, A.; Parker, D. H., Velocity map imaging of ions and electrons using electrostatic lenses: Application in photoelectron and photofragment ion imaging of molecular oxygen. Rev. Sci. Instrum. 1997, 68, 3477-3484.

35. Weichman, M. L.; DeVine, J. A.; Levine, D. S.; Kim, J. B.; Neumark, D. M., Isomer-specific vibronic structure of the 9-, 1-, and 2-anthracenyl radicals via slow photoelectron velocity-map imaging. PNAS 2016, 113, 1698-1705.

36. Chandler, D. W.; Houston, P. L., Two-dimensional Imaging of State-selected Photodissociation Products Detected by Multiphoton Ionization. J. Chem. Phys. 1987, 87, 1445-1447. 
37. Doyle, M. B.; Abeyasera, C.; Suits, A. G. NuACQ.

http://faculty.missouri.edu/suitsa/NuAqc.html.

38. Bosenberg, W. R.; Guyer, D. R., Broadly Tunable, Single-frequency Optical Parametric

Frequency-conversion System. J. Opt. Soc. Am. B-Opt. Phys. 1993, 10, 1716-1722.

39. Dick, B., Inverting ion images without Abel inversion: maximum entropy reconstruction of velocity maps. Phys. Chem. Chem. Phys. 2014, 16, 570-580.

40. Blondel, C.; Chaibi, W.; Delsart, C.; Drag, C.; Goldfarb, F.; Kroger, S., The electron affinities of $\mathrm{O}, \mathrm{Si}$, and $\mathrm{S}$ revisited with the photodetachment microscope. Eur. Phys. J. D 2005, 33, 335-342.

41. Kitsopoulos, T. N.; Waller, I. M.; Loeser, J. G.; Neumark, D. M., High Resolution Threshold Photodetachment Spectroscopy Of Negative Ions. Chem. Phys. Lett. 1989, 159, 300-306.

42. Wolk, A. B.; Leavitt, C. M.; Garand, E.; Johnson, M. A., Cryogenic Ion Chemistry and Spectroscopy. Accts. Chem. Res. 2014, 47, 202-210.

43. Schwarz, H.; Asmis, K. R., Identification of Active Sites and Structural Characterization of Reactive Ionic Intermediates by Cryogenic Ion Trap Vibrational Spectroscopy. Chem. Eur. J. 2019, 25, 2112-2126.

44. DeVine, J. A.; Weichman, M. L.; Laws, B.; Chang, J.; Babin, M. C.; Balerdi, G.; Xie, C. J.; Malbon, C. L.; Lineberger, W. C.; Yarkony, D. R., et al., Encoding of vinylidene isomerization in its anion photoelectron spectrum. Science 2017, 358, 336-339.

45. Stanton, J. F.; Gauss, J., Vibrational structure in the vinylidene anion photoelectron spectrum: Closing the gap between theory and experiment. J. Chem. Phys. 1999, 110, 6079-6080. 
TABLE I. Peak positions, assignments, and calculated energies for detachment transitions for the cryoSEVI and IR cryo-SEVI spectra of the $\widetilde{X}^{2} \Pi \leftarrow \tilde{X}^{1} \Sigma^{+}$photodetachment of $\mathrm{OH}^{-}$in Figure 1 . Transitions from vibrationally-excited anions are shown in red. All values are reported in $\mathrm{cm}^{-1}$ and uncertainties in peak positions correspond to one standard deviation obtained from a Gaussian fit to the high-resolution scan of the experimental peak.

\begin{tabular}{ccccc}
\hline \hline Label & eBE & $\begin{array}{c}\text { Vibrational } \\
\text { Transition }\end{array}$ & $\begin{array}{c}\text { Rotational } \\
\text { Transition }\end{array}$ & Reported Energy \\
\hline $\mathrm{A}_{3}$ & $14568(4)$ & $0-0$ & $\mathrm{P} 3(4)$ & 14568.98 \\
$\mathrm{~B}_{3}$ & $14600(4)$ & $0-0$ & $\mathrm{P} 3(3)$ & 14600.18 \\
$\mathrm{C}_{3}$ & $14628(3)$ & $0-0$ & $\mathrm{P} 3(2)$ & 14628.64 \\
$\mathrm{D}_{3}$ & $14704(1)$ & $0-0$ & $\mathrm{Q} 3(1)$ & 14703.54 \\
$\mathrm{E}_{3}$ & $14713(1)$ & $0-0$ & $\mathrm{Q} 3(2)$ & 14712.36 \\
$\mathrm{~d}_{3}$ & $14718(1)$ & $1-1$ & $\mathrm{Q} 3(1)$ & 14717.93 \\
$\mathrm{~F}_{3}$ & $14718(2)$ & $0-0$ & $\mathrm{Q} 3(3)$ & 14718.37 \\
$\mathrm{G}_{3}$ & $14741(2)$ & $0-0$ & $\mathrm{R} 3(0)$ & 14740.98 \\
$\mathrm{H}_{3}$ & $14787(1)$ & $0-0$ & $\mathrm{R} 3(1)$ & 14787.26 \\
$\mathrm{~h}_{3}$ & $14799(1)$ & $1-1$ & $\mathrm{R} 3(1)$ & 14798.66 \\
$\mathrm{~A}_{1}$ & $14809(3)$ & $0-0$ & $\mathrm{Q} 1(3)$ & 14805.51 \\
$\mathrm{~B}_{1}$ & $14816(2)$ & $0-0$ & $\mathrm{Q} 1(2)$ & 14816.39 \\
$\mathrm{C}_{1}$ & $14830(1)$ & $0-0$ & $\mathrm{Q} 1(1)$ & 14829.99 \\
$\mathrm{c}_{1}$ & $14845(1)$ & $1-1$ & $\mathrm{Q} 1(1)$ & 14844.82 \\
$\mathrm{D}_{1}$ & $14868(2)$ & $0-0$ & $\mathrm{R} 1(0)$ & 14867.43 \\
$\mathrm{E}_{1}$ & $14891(1)$ & $0-0$ & $\mathrm{R} 1(1)$ & 14891.29 \\
$\mathrm{e}_{1}$ & $14904(2)$ & $1-1$ & $\mathrm{R} 1(1)$ & 14903.50 \\
$\mathrm{~F}_{1}$ & $14918(2)$ & $0-0$ & $\mathrm{R} 1(2)$ & 14917.69 \\
$\mathrm{G}_{1}$ & $14929(4)$ & $0-0$ & $\mathrm{~S} 1(0)$ & 14928.74 \\
$\mathrm{H}_{1}$ & $14945(2)$ & $0-0$ & $\mathrm{R} 1(3)$ & 14945.97 \\
$\mathrm{I}_{1}$ & $14993(2)$ & $0-0$ & $\mathrm{~S} 1(1)$ & 14992.58 \\
$\mathrm{i}_{1}$ & $15001(2)$ & $1-1$ & $\mathrm{~S} 1(1)$ & 15000.67 \\
\hline \hline
\end{tabular}

*Reported transition energies were calculated based on the experimental values of R3(0) and R1(0), reported by Goldfarb, ${ }^{10}$ and the expected shifts in energy based on rotational energy level spacings reported by Rosenbaum and Mélen. ${ }^{21,29}$ 


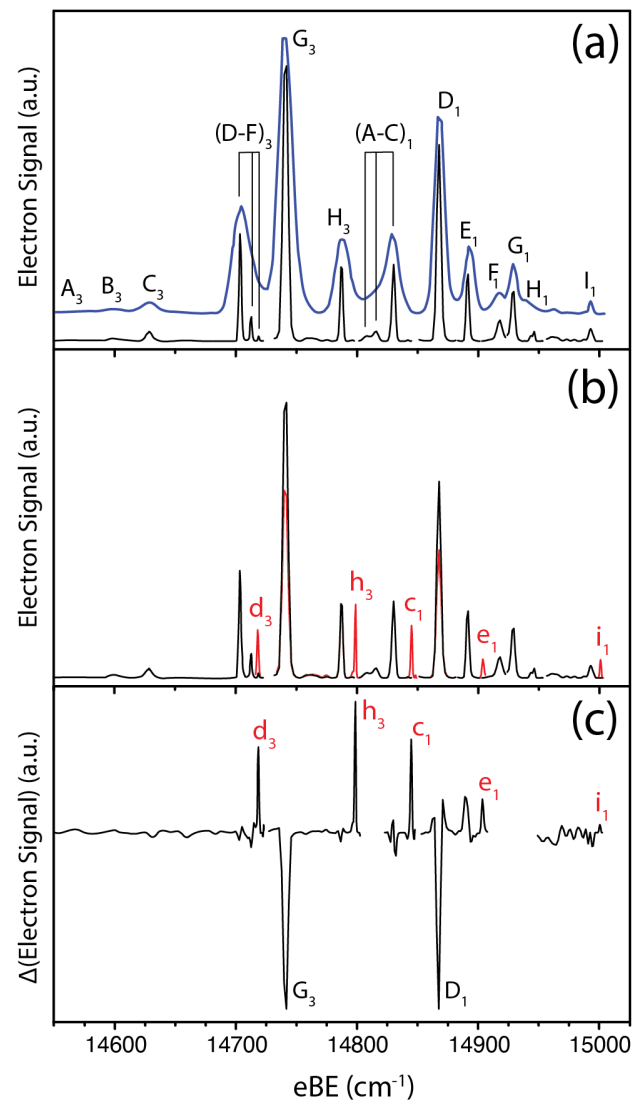

FIG. 1. Cryo-SEVI spectrum (a), IR cryo-SEVI (b), and difference spectrum (c) of the $\tilde{X}^{2} \Pi \leftarrow \tilde{X}^{1} \Sigma^{+}$ detachment of $\mathrm{OH}^{-}$. The blue trace is an overview spectrum taken with a photon energy of $15004.07 \mathrm{~cm}^{-1}$, while the black traces are high-resolution scans taken near threshold, and the red traces are highresolution scans that show new or depleted features after IR pre-excitation. Subscripts of 3 and 1 indicate transitions to $\mathrm{OH}$ levels with $\Omega^{\prime}=3 / 2$ and $\Omega^{\prime}=1 / 2$. respectively. 


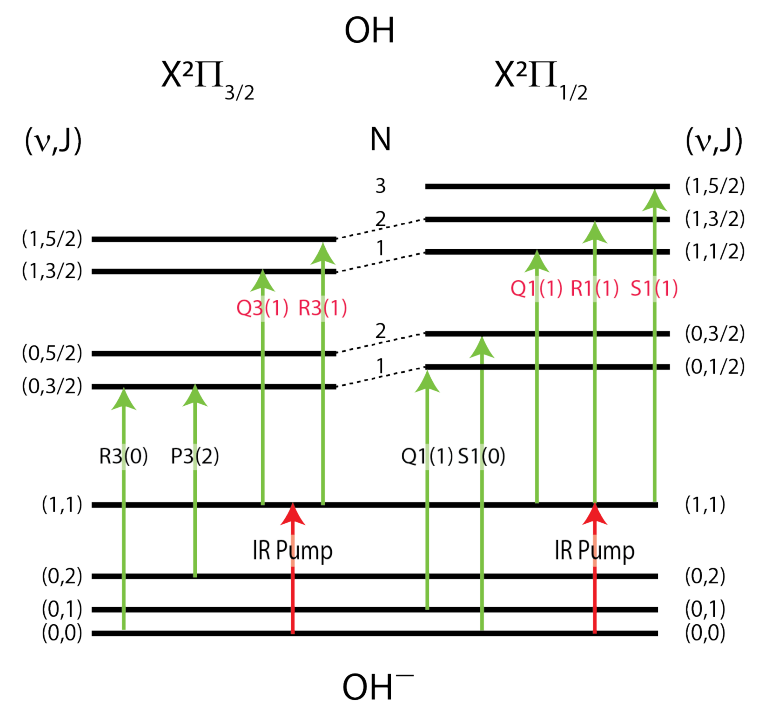

FIG. 2. Relevant rovibrational energy levels of $\mathrm{OH}^{-}$and $\mathrm{OH}$. Arrows depict some experimentally observed transitions originating from the $v "=0,1$ levels of $\mathrm{OH}^{-}$. 

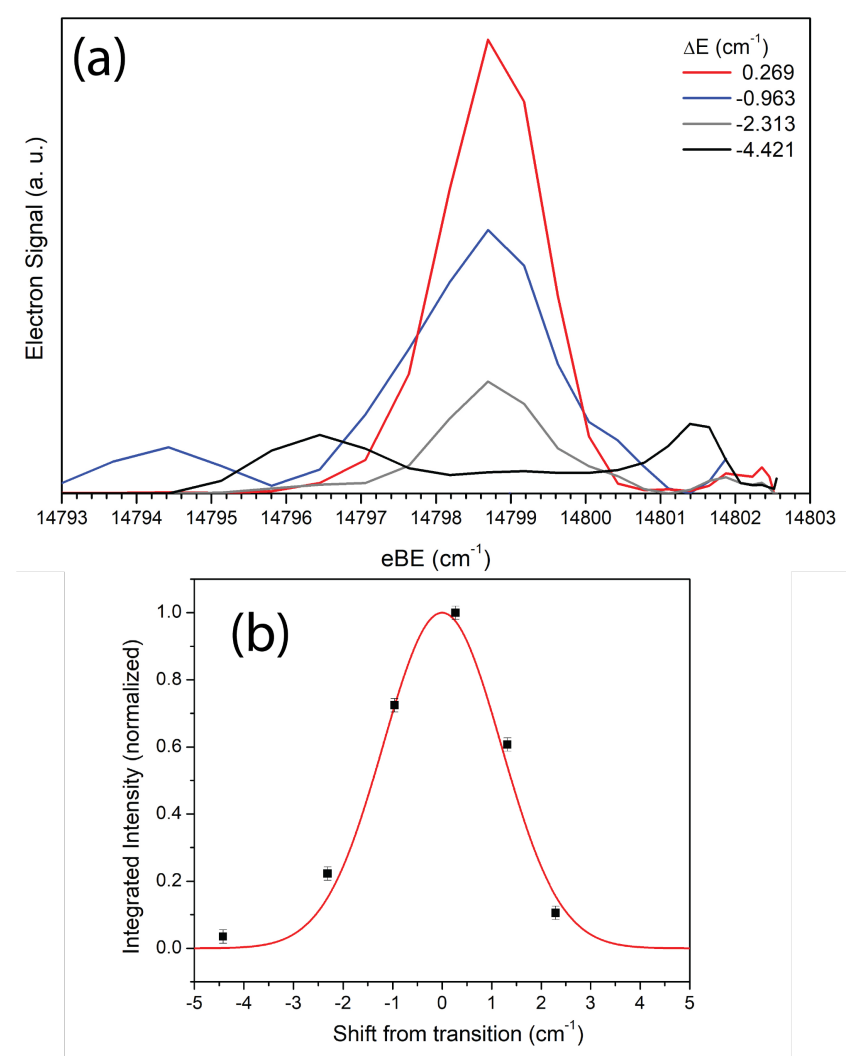

FIG. 3. (a) Representative IR cryo-SEVI spectra of feature $h_{3}$ taken with a fixed detachment frequency ( $\mathrm{h} v=14802.5 \mathrm{~cm}^{-1}$ ) while the IR wavelength is scanned across the $\mathrm{R}(0)$ transition of $\mathrm{OH}^{-}$. (b) Plot of integrated intensity of $h_{3}$ as a function of IR wavelength (black squares) and fit to Gaussian (red trace). The integrated intensity is normalized to the most intense transition measured. 